\title{
Evaluation of Maize Genotypes under Rice Fallow Situation
}

\author{
M. Paramesh ${ }^{1 *}$, K.H.P. Reddy ${ }^{1}$, D.M. Reddy ${ }^{1}$, V. Rajarajeswari ${ }^{2}$ and \\ B.V. Bhaskara Reddy \\ ${ }^{1}$ Department of Genetics and Plant Breeding, S.V. Agricultural College, \\ Tirupati- 517502, India \\ ${ }^{2}$ Department of Crop Physiology, S.V. Agricultural College, Tirupati- 517502, India \\ ${ }^{3}$ Department of Plant Pathology, RARS, Tirupati- 517502, India
}

\section{A B S T R A C T}

\begin{tabular}{|l|}
\hline Ke y w or d s \\
Rice fallow maize, Mean \\
performance, Hybrids
\end{tabular}

Introduction

Maize is the third most important cereal crop after rice and wheat and referred as 'Queen of cereals' due to its high yield potential among cereals. Maize is cultivated in an area of 9.63 million hectares, with a production of 25.90 million tonnes and with the productivity of $2689 \mathrm{~kg} \mathrm{ha}^{-1}$ in India (Indiastat.com, 2016-17). While, in Andhra Pradesh it is grown in an area of 2.50 lakh hectares, with a production of 16.53 lakh tonnes and with the productivity of $6604 \mathrm{~kg} \mathrm{ha}^{-1}$ (Directorate of Economics \& Statistics, Govt. of Andhra Pradesh, 2016-17).

Through exploitation of single cross hybrids in maize, the production and productivity has been increased significantly in traditional areas and different cropping systems. Still there is a dramatic increase of maize demand in our country and the current trend appears to be unable to keep pace. Since, the option of further increasing the maize area is limited, the viable option left is increasing the production and productivity by growing hybrid maize in different cropping systems. Earlier rice-pulse system was adopted by the farmers especially in Godavari, Krishna and Guntur districts of Andhra Pradesh. But pulses productivity has decreased due to the incidence of yellow mosaic and leaf curl viruses. Hence, instead of pulses, irrigated dry crops like sunflower and maize were recommended but farmers are not willing to 
take up because lot of time and labour are required to make the soil suitable for sowing. Hence, Zero tillage (No till) technology has come into practice which not only has short term benefits like saving of 25-30\% of energy for field preparation, advancement in sowings by 20-25 days but also long term benefits like improvement in the organic carbon content of the soil, checking soil erosion etc. Due to higher productivity, profitability and assured alternative rabi crop after rice, acreage of maize has shown an increasing trend and ricemaize emerged as a potential cropping system. No-till maize in rice fallows demonstrated a potential benefit of saving on cost of production ranging from Rs.3,800-5,500/ha. This system was mainly followed in coastal districts of Andhra Pradesh and Warangal and Khammam districts of Telangana states. But rice fallow maize trend was slowly acquiring its pace throughout the districts of Andhra Pradesh and Telangana.

To overcome the agronomic and soil management constraints in productivity of maize in rice fallows, development of right type of genotypes appears to be the only alternative. Hence, an attempt was made to understand the mechanism of adaptation to such conditions and studying its genetics and breeding to improve maize for rice based cropping systems.

\section{Materials and Methods}

The experimental material for this study comprised of eleven inbred lines viz., BML 51, BML 5, CM 105, BML 2, BML 6, BML 7, BML 15, BML 14, CM 118, CM 119 and CML 124 and their $55 \mathrm{~F}_{1} \mathrm{~s}$ derived by crossing in $11 \times 11$ half diallel fashion. Eleven parents and $55 \mathrm{~F}_{1} \mathrm{~s}$ were evaluated in randomized block design with three replications at Sri Venkateswara Agricultural College Farm, Tirupati, Andhra Pradesh in rabi, 2016-17. The plot size for each entry was one row of five meter length, with a spacing of $60 \mathrm{~cm}$ and $20 \mathrm{~cm}$ between row to row and plant to plant, respectively. In rice fallow system, maize seeds were sown at optimum moisture condition immediately after harvesting of the kharif rice crop. The maize seeds were sown by dibbling method with the help of a wooden peg. The recommended dose of fertilizers used were same as normal rabi maize (120:80:60 $\mathrm{kg} \mathrm{ha}^{-1}$ ), but the first dose of fertilizers applied immediately after sowing by making a furrow parallel and $5 \mathrm{~cm}$ apart from seeds sown. For weed management atrazine @ $2.5 \mathrm{~kg}$ and paraquate@ 2.5 lt per hectare were mixed in 200 lt of water and sprayed on same day after sowing. The remaining agronomical practices followed were similar to normal maize cultivation.

The observations on seventeen yield and yield component traits viz., days to 50 per cent tasseling, days to 50 per cent silking, anthesissilking interval, days to maturity, SPAD chlorophyll meter reading (SCMR), specific leaf area, relative water content, leaf area index, plant height, tassel length, ear length, ear girth, number of kernel rows per ear, number of kernels per ear row, 100 kernel weight, protein content and kernel yield per plant were recorded on five randomly tagged competitive plants in each genotype in each replication. The mean of these five plants were used in the statistical analysis.

However, for days to $50 \%$ tasseling, days to $50 \%$ silking, anthesis-silking interval and days to maturity the observations were recorded on plot basis. The data were subjected to analysis of variance as per the method suggested by Panse and Sukhatme (1985).

\section{Results and Discussion}

The analysis of variance of the parents and the hybrids for yield and yield component traits are presented in Table 1 and 2. 
Table.1 Analysis of variance for seventeen quantitative traits in eleven parents of maize under rice fallow situation

\begin{tabular}{|c|c|c|c|c|}
\hline \multirow{2}{*}{$\begin{array}{l}\text { S. } \\
\text { No. }\end{array}$} & \multirow[t]{2}{*}{ Characters } & \multicolumn{3}{|c|}{ Mean sum of squares } \\
\hline & & $\begin{array}{c}\text { Replications } \\
\text { (df: } 2)\end{array}$ & Parents (df: 10) & $\begin{array}{c}\text { Error } \\
\text { (df: } 20)\end{array}$ \\
\hline 1. & Days to $50 \%$ tasseling & 1.12 & $11.12^{* *}$ & 0.32 \\
\hline 2. & Days to $50 \%$ silking & 2.55 & $18.79 * *$ & 0.88 \\
\hline 3. & Anthesis silking interval & 0.39 & $1.93 * *$ & 0.26 \\
\hline 4. & Days to maturity & 1.49 & $35.03 * *$ & 0.79 \\
\hline 5. & SPAD Chlorophyll Meter Reading & 0.57 & $49.39 * *$ & 1.80 \\
\hline 6. & Specific leaf area $\left(\mathrm{cm}^{2} \mathrm{~g}^{-1}\right)$ & 133.03 & $644.04 * *$ & 79.77 \\
\hline 7. & Relative water content (\%) & 0.53 & $36.20 * *$ & 7.14 \\
\hline 8. & Leaf area index & 0.02 & $0.72 * *$ & 0.02 \\
\hline 9. & Plant height $(\mathrm{cm})$ & 81.95 & $1654.73 * *$ & 66.34 \\
\hline 10. & Tassel length $(\mathrm{cm})$ & 11.90 & $47.51^{* *}$ & 2.81 \\
\hline 11. & Ear length $(\mathrm{cm})$ & 0.10 & $15.08^{* *}$ & 0.70 \\
\hline 12. & Ear girth $(\mathrm{cm})$ & 0.16 & $3.20 * *$ & 0.56 \\
\hline 13. & No. of kernel rows/ear & 1.34 & $3.03 * *$ & 0.38 \\
\hline 14. & No. of kernels/ear row & 1.43 & $41.51^{* *}$ & 1.10 \\
\hline 15. & 100 kernel weight $(\mathrm{g})$ & 0.98 & $55.53 * *$ & 1.02 \\
\hline 16. & Protein content $(\%)$ & 0.01 & $1.80 * *$ & 0.01 \\
\hline 17. & Kernel yield per plant (g) & 5.85 & $771.08 * *$ & 13.55 \\
\hline
\end{tabular}

* Significant at $5 \%$ level; ** Significant at $1 \%$ level

Table.2 Analysis of variance for seventeen quantitative traits in fifty five hybrids of maize under rice fallow situation

\begin{tabular}{|c|c|c|c|c|}
\hline \multirow{2}{*}{$\begin{array}{l}\text { S. } \\
\text { No. }\end{array}$} & \multirow[t]{2}{*}{ Characters } & \multicolumn{3}{|c|}{ Mean sum of squares } \\
\hline & & $\begin{array}{l}\text { Replications } \\
\text { (df: } 2 \text { ) }\end{array}$ & $\begin{array}{c}\text { Hybrids } \\
\text { (df: 54) }\end{array}$ & $\begin{array}{c}\text { Error } \\
\text { (df: 108) }\end{array}$ \\
\hline 1. & Days to $50 \%$ tasseling & 9.02 & $4.58 * *$ & 0.83 \\
\hline 2. & Days to $50 \%$ silking & 10.15 & $6.24 * *$ & 1.34 \\
\hline 3. & Anthesis silking interval & 0.26 & $0.59 *$ & 0.42 \\
\hline 4. & Days to maturity & 11.25 & $14.10^{* *}$ & 0.84 \\
\hline 5. & SPAD Chlorophyll Meter Reading & 33.35 & $37.21 * *$ & 4.82 \\
\hline 6. & Specific leaf area $\left(\mathrm{cm}^{2} \mathrm{~g}^{-1}\right)$ & 136.56 & $434.61 * *$ & 150.50 \\
\hline 7. & Relative water content $(\%)$ & 139.84 & $46.46^{* *}$ & 8.96 \\
\hline 8. & Leaf area index & 0.09 & $1.23 * *$ & 0.01 \\
\hline 9. & Plant height $(\mathrm{cm})$ & 528.89 & $673.95 * *$ & 88.65 \\
\hline 10. & Tassel length $(\mathrm{cm})$ & 8.60 & $16.77 * *$ & 6.44 \\
\hline 11. & Ear length $(\mathrm{cm})$ & 1.91 & $5.00^{* * *}$ & 0.86 \\
\hline 12. & Ear girth $(\mathrm{cm})$ & 1.28 & $0.86^{* *}$ & 0.31 \\
\hline 13. & No. of kernel rows/ear & 1.45 & $1.42 * *$ & 0.43 \\
\hline 14. & No. of kernels/ear row & 22.23 & $37.69 * *$ & 3.90 \\
\hline 15. & 100 kernel weight $(\mathrm{g})$ & 0.68 & $20.26^{* *}$ & 0.96 \\
\hline 16. & Protein content $(\%)$ & 0.45 & $2.44 * *$ & 0.01 \\
\hline 17. & Kernel yield per plant (g) & 73.32 & $629.17 * *$ & 19.85 \\
\hline
\end{tabular}

* Significant at $5 \%$ level; ** Significant at $1 \%$ level 
Table.3 Mean performance of eleven parents of maize for seventeen quantitative characters under rice fallow situation

\begin{tabular}{|c|c|c|c|c|c|c|c|c|c|c|c|c|c|c|c|c|c|c|}
\hline S. No. & Parent & DFT & DFS & ASI & DM & SCMR & SLA & RWC & LAI & PH & TL & EL & EG & $\mathrm{KR} / \mathbf{E}$ & K/ER & $100 \mathrm{KW}$ & PC & KYD \\
\hline 1 & BML 51 & 71.00 & 73.00 & 2.00 & 103.00 & 48.80 & 161.11 & 86.42 & 1.90 & 174.87 & 32.93 & 16.27 & 13.00 & 11.87 & 23.00 & 27.16 & 8.50 & 70.68 \\
\hline 2 & BML 5 & 70.33 & 73.00 & 2.67 & 104.33 & 41.00 & 160.15 & 78.25 & 1.32 & 188.27 & 35.33 & 18.27 & 13.07 & 12.40 & 21.60 & 19.64 & 8.56 & 55.55 \\
\hline 3 & CM 105 & 67.67 & 69.67 & 2.00 & 105.00 & 45.97 & 170.60 & 82.22 & 1.57 & 174.87 & 35.27 & 17.27 & 13.40 & 12.13 & 23.73 & 23.76 & 8.47 & 59.37 \\
\hline 4 & BML 2 & 67.67 & 70.33 & 2.67 & 108.67 & 52.63 & 180.94 & 86.02 & 2.20 & 208.67 & 43.20 & 19.47 & 14.40 & 13.47 & 28.80 & 30.56 & 9.18 & 90.51 \\
\hline 5 & BML 6 & 69.67 & 72.67 & 3.00 & 108.33 & 51.83 & 191.12 & 86.01 & 2.09 & 195.33 & 40.93 & 18.53 & 14.67 & 14.00 & 25.60 & 26.36 & 8.50 & 83.55 \\
\hline 6 & BML 7 & 72.67 & 77.33 & 4.67 & 110.00 & 51.57 & 188.36 & 88.61 & 1.89 & 177.87 & 37.00 & 15.67 & 13.33 & 12.00 & 21.27 & 26.63 & 8.21 & 64.00 \\
\hline 7 & BML 15 & 70.67 & 72.67 & 2.00 & 108.33 & 46.53 & 162.09 & 83.24 & 1.09 & 192.80 & 35.07 & 17.60 & 14.07 & 13.73 & 27.13 & 22.08 & 7.91 & 81.60 \\
\hline 8 & BML 14 & 67.67 & 70.00 & 2.33 & 101.67 & 54.00 & 197.82 & 85.59 & 2.79 & 194.47 & 41.47 & 19.40 & 14.33 & 14.13 & 25.47 & 28.92 & 7.66 & 87.95 \\
\hline 9 & CM 118 & 67.67 & 69.67 & 2.00 & 100.67 & 52.10 & 193.42 & 83.04 & 1.53 & 119.53 & 29.40 & 13.47 & 12.13 & 12.40 & 19.40 & 17.03 & 7.78 & 48.64 \\
\hline 10 & CM 119 & 68.33 & 71.00 & 2.67 & 100.33 & 54.23 & 193.85 & 91.52 & 1.27 & 163.73 & 36.67 & 14.60 & 12.40 & 13.33 & 18.47 & 19.42 & 9.56 & 47.70 \\
\hline \multirow[t]{7}{*}{11} & CML 124 & 72.33 & 75.67 & 3.33 & 105.00 & 48.05 & 169.30 & 84.24 & 1.91 & 171.73 & 37.93 & 13.20 & 11.40 & 11.07 & 17.00 & 26.07 & 10.18 & 53.80 \\
\hline & Grand mean & 69.61 & 72.27 & 2.67 & 105.03 & 49.70 & 178.98 & 85.01 & 1.78 & 178.38 & 36.84 & 16.70 & 13.29 & 12.78 & 22.86 & 24.33 & 8.59 & 67.58 \\
\hline & Range (Min.) & 67.67 & 69.67 & 2.00 & 100.33 & 41.00 & 160.15 & 78.25 & 1.09 & 119.53 & 29.40 & 13.20 & 11.40 & 11.07 & 17.00 & 17.03 & 7.66 & 47.70 \\
\hline & Range (Max.) & 72.67 & 77.33 & 4.67 & 110.00 & 54.23 & 197.82 & 91.52 & 2.79 & 208.67 & 43.20 & 19.47 & 14.67 & 14.13 & 28.80 & 30.56 & 10.18 & 90.51 \\
\hline & C.D. & 0.97 & 1.61 & 0.88 & 1.52 & 2.30 & 15.32 & 4.58 & 0.22 & 13.97 & 2.88 & 1.43 & 1.28 & 1.04 & 1.80 & 1.74 & 0.24 & 6.31 \\
\hline & $\mathrm{S} . \mathrm{E}(\mathrm{m})$ & 0.33 & 0.54 & 0.30 & 0.51 & 0.78 & 5.16 & 1.54 & 0.07 & 4.70 & 0.97 & 0.48 & 0.43 & 0.35 & 0.61 & 0.59 & 0.08 & 2.13 \\
\hline & C.V. & 0.81 & 1.30 & 19.14 & 0.84 & 2.70 & 4.99 & 3.14 & 7.17 & 4.57 & 4.55 & 4.98 & 5.61 & 4.74 & 4.59 & 4.16 & 1.61 & 5.45 \\
\hline
\end{tabular}

DFT - Days to 50\% Tasseling, DFS - Days to 50\% Silking, ASI - Anthesis Silking Interval, DM - Days to Maturity, SCMR - SPAD Chlorophyll Meter Reading SLA - Specific Leaf Area $\left(\mathrm{cm}^{2} \mathrm{~g}^{-1}\right)$, RWC - Relative Water Content (\%), LAI - Leaf Area Index, PH - Plant Height ( $\left.\mathrm{cm}\right)$, TL - Tassel Length (cm), EL - Ear Length (cm), EG - Ear girth (cm), KR/E - No. of Kernel Rows per Ear, K/ER - No. of Kernels per Ear Row, 100 KW - 100 Kernel Weight (g), PC Protein Content (\%), KYD - Kernel Yield per Plant (g) 
Table.4 Mean performance of fifty five hybrids of maize for seventeen quantitative characters under rice fallow situation

\begin{tabular}{|c|c|c|c|c|c|c|c|c|c|c|c|c|c|c|c|c|c|c|}
\hline S. No. & Hybrid & DFT & DFS & ASI & DM & SCMR & SLA & RWC & LAI & PH & TL & EL & EG & KR/E & K/ER & $100 \mathrm{KW}$ & PC & KYD \\
\hline 1 & BML $51 \times$ BML 5 & 71.33 & 73.67 & 2.33 & 107.33 & 49.20 & 180.17 & 88.46 & 2.45 & 205.33 & 40.07 & 19.33 & 14.27 & 12.93 & 24.87 & 25.69 & 10.34 & 98.36 \\
\hline$\overline{2}$ & BML $51 \times$ CM 105 & 66.67 & 69.33 & 2.67 & 103.33 & 53.80 & 176.16 & 89.46 & 2.80 & 187.73 & 38.47 & 17.93 & 13.33 & 12.13 & 24.73 & 24.19 & 8.66 & 93.54 \\
\hline 3 & BML $51 \times$ BML 2 & 65.00 & 67.33 & 2.33 & 103.00 & 50.53 & 193.48 & 85.14 & 1.79 & 231.53 & 40.47 & 20.33 & 14.47 & 12.80 & 32.27 & 28.72 & 10.41 & 108.19 \\
\hline 4 & BML $51 \times$ BML 6 & 67.33 & 69.33 & 2.00 & 102.67 & 52.07 & 173.48 & 90.26 & 2.28 & 220.13 & 39.13 & 18.53 & 14.33 & 13.87 & 32.20 & 30.19 & 8.97 & 109.54 \\
\hline 5 & BML $51 \times$ BML 7 & 68.67 & 70.67 & 2.00 & 100.67 & 54.80 & 166.41 & 83.85 & 1.61 & 206.93 & 42.40 & 20.20 & 15.80 & 14.27 & 31.13 & 31.84 & 8.85 & 120.14 \\
\hline 6 & BML $51 \times$ BML 15 & 69.33 & 71.67 & 2.33 & 103.33 & 50.20 & 159.85 & 84.82 & 2.49 & 221.40 & 39.20 & 19.87 & 14.07 & 12.93 & 31.73 & 22.21 & 8.85 & 102.72 \\
\hline 7 & BML $51 \times$ BML 14 & 69.00 & 71.00 & 2.00 & 106.67 & 51.13 & 175.29 & 84.15 & 2.46 & 212.80 & 40.60 & 18.80 & 14.53 & 12.67 & 26.93 & 30.23 & 8.65 & 96.33 \\
\hline 8 & BML $51 \times$ CM 118 & 68.00 & 70.00 & 2.00 & 106.67 & 52.20 & 161.49 & 86.11 & 3.07 & 235.73 & 40.00 & 19.87 & 14.73 & 13.73 & 30.07 & 31.62 & 9.64 & 104.95 \\
\hline 9 & BML $51 \times$ CM 119 & 67.67 & 70.00 & 2.33 & 103.33 & 53.87 & 172.47 & 86.62 & 2.79 & 224.27 & 42.27 & 16.67 & 14.00 & 13.33 & 23.47 & 26.24 & 9.07 & 87.07 \\
\hline 10 & BML $51 \times$ CML 124 & 69.33 & 71.33 & 2.00 & 103.00 & 55.80 & 182.58 & 79.80 & 1.13 & 218.40 & 38.20 & 19.40 & 14.67 & 13.20 & 30.33 & 27.76 & 8.34 & 103.70 \\
\hline 11 & BML $5 \times$ CM 105 & 67.33 & 69.33 & 2.00 & 104.00 & 53.47 & 160.33 & 86.94 & 2.11 & 199.53 & 35.13 & 19.67 & 15.40 & 14.13 & 29.93 & 31.07 & 9.52 & 123.31 \\
\hline 12 & BML $5 \times$ BML 2 & 69.00 & 72.00 & 3.00 & 103.00 & 52.30 & 178.00 & 86.26 & 1.47 & 209.73 & 41.67 & 20.93 & 14.60 & 12.67 & 32.20 & 28.29 & 8.73 & 108.28 \\
\hline 13 & BML $5 \times$ BML 6 & 69.67 & 73.00 & 3.33 & 103.33 & 45.23 & 187.72 & 90.79 & 2.73 & 229.33 & 37.80 & 20.40 & 15.27 & 13.73 & 33.87 & 27.26 & 9.46 & 110.11 \\
\hline 14 & BML $5 \times$ BML 7 & 68.00 & 70.67 & 2.67 & 99.33 & 50.50 & 172.83 & 77.67 & 2.83 & 231.33 & 43.07 & 20.60 & 14.67 & 13.87 & 34.27 & 28.76 & 8.54 & 121.32 \\
\hline 15 & BML $5 \times$ BML 15 & 69.33 & 71.67 & 2.33 & 98.67 & 48.23 & 182.72 & 86.43 & 1.50 & 221.67 & 39.87 & 20.73 & 14.67 & 12.53 & 35.87 & 29.50 & 9.74 & 118.75 \\
\hline 16 & BML $5 \times$ BML 14 & 65.00 & 67.33 & 2.33 & 101.33 & 45.80 & 166.20 & 83.97 & 2.54 & 220.33 & 45.47 & 20.73 & 14.60 & 12.80 & 32.87 & 29.91 & 10.73 & 109.39 \\
\hline 17 & BML $5 \times$ CM 118 & 67.33 & 69.33 & 2.00 & 104.00 & 48.67 & 169.20 & 71.03 & 1.11 & 199.77 & 39.20 & 19.60 & 14.53 & 14.13 & 21.13 & 19.77 & 8.15 & 79.34 \\
\hline 18 & BML $5 \times$ CM 119 & 69.00 & 71.00 & 2.00 & 102.33 & 50.47 & 186.17 & 82.43 & 2.18 & 225.07 & 44.40 & 19.13 & 14.13 & 13.60 & 28.53 & 27.26 & 10.66 & 98.72 \\
\hline 19 & BML $5 \times$ CML 124 & 69.33 & 72.00 & 2.67 & 104.00 & 42.67 & 173.94 & 85.62 & 3.03 & 212.93 & 42.07 & 19.33 & 14.60 & 13.47 & 33.00 & 27.10 & 10.33 & 103.62 \\
\hline 20 & CM $105 \times$ BML 2 & 68.67 & 71.00 & 2.33 & 102.67 & 43.20 & 162.53 & 90.35 & 1.23 & 186.20 & 36.67 & 17.93 & 14.40 & 12.27 & 28.73 & 24.12 & 8.54 & 92.21 \\
\hline 21 & CM $105 \times$ BML 6 & 67.67 & 70.67 & 3.00 & 106.33 & 49.63 & 160.95 & 86.46 & 2.29 & 204.27 & 38.53 & 17.07 & 14.53 & 14.53 & 26.93 & 23.40 & 8.33 & 89.44 \\
\hline 22 & CM $105 \times$ BML 7 & 68.67 & 71.33 & 2.67 & 105.67 & 50.27 & 168.46 & 81.62 & 2.20 & 202.87 & 41.73 & 17.27 & 13.93 & 13.20 & 22.00 & 26.02 & 7.89 & 76.17 \\
\hline 23 & CM $105 \times$ BML 15 & 68.67 & 71.33 & 2.67 & 102.00 & 38.91 & 174.57 & 86.68 & 1.85 & 211.93 & 35.20 & 18.80 & 15.27 & 14.53 & 31.53 & 24.17 & 8.93 & 108.69 \\
\hline 24 & CM $105 \times$ BML 14 & 67.33 & 69.33 & 2.00 & 100.33 & 48.60 & 165.62 & 89.99 & 3.21 & 204.60 & 42.60 & 17.47 & 15.07 & 14.40 & 26.27 & 27.95 & 7.99 & 95.20 \\
\hline 25 & CM $105 \times$ CM 118 & 68.00 & 70.00 & 2.00 & 102.33 & 52.00 & 190.45 & 85.73 & 1.93 & 202.87 & 39.40 & 17.40 & 14.27 & 13.87 & 27.07 & 28.07 & 7.87 & 95.74 \\
\hline 26 & CM $105 \times$ CM 119 & 68.67 & 71.67 & 3.00 & 103.67 & 48.57 & 186.55 & 83.68 & 3.53 & 207.33 & 40.00 & 18.27 & 15.07 & 13.33 & 28.87 & 29.03 & 7.78 & 98.37 \\
\hline 27 & CM $105 \times$ CML 124 & 67.67 & 70.00 & 2.33 & 103.00 & 47.93 & 169.76 & 84.90 & 2.78 & 213.73 & 39.07 & 16.87 & 14.47 & 13.33 & 28.31 & 29.61 & 8.38 & 104.36 \\
\hline 28 & BML $2 \times$ BML 6 & 66.33 & 68.33 & 2.00 & 100.00 & 50.83 & 182.63 & 82.83 & 2.56 & 192.13 & 40.87 & 17.60 & 14.53 & 13.87 & 28.33 & 26.27 & 8.63 & 97.08 \\
\hline 29 & BML $2 \times$ BML 7 & 69.00 & 72.33 & 3.33 & 100.33 & 49.17 & 205.75 & 90.66 & 2.17 & 227.37 & 41.53 & 20.13 & 14.60 & 13.87 & 34.33 & 31.45 & 7.54 & 138.31 \\
\hline$\overline{30}$ & BML $2 \times$ BML 15 & 68.00 & 70.33 & 2.33 & 99.33 & 51.17 & 182.00 & 82.96 & 2.31 & 222.80 & 37.00 & 19.53 & 14.47 & 12.93 & 33.13 & 30.63 & 9.58 & 112.34 \\
\hline
\end{tabular}


Cont...

\begin{tabular}{|c|c|c|c|c|c|c|c|c|c|c|c|c|c|c|c|c|c|c|}
\hline S. No. & Hybrid & DFT & DFS & ASI & DM & SCMR & SLA & RWC & LAI & PH & TL & EL & EG & KR/E & K/ER & $100 \mathrm{KW}$ & PC & KYD \\
\hline 31 & BML $2 \times$ BML 14 & 67.33 & 69.67 & 2.33 & 101.33 & 47.37 & 197.61 & 87.18 & 2.23 & 194.80 & 36.93 & 17.80 & 13.33 & 13.60 & 29.67 & 27.61 & 9.51 & 105.33 \\
\hline 32 & BML $2 \times$ CM 118 & 66.67 & 69.33 & 2.67 & 102.67 & 53.27 & 201.31 & 86.63 & 1.73 & 205.27 & 40.00 & 17.40 & 13.47 & 13.47 & 26.27 & 24.97 & 9.08 & 89.30 \\
\hline 33 & BML $2 \times$ CM 119 & 66.00 & 68.00 & 2.00 & 100.00 & 46.70 & 173.94 & 86.95 & 3.37 & 214.53 & 42.93 & 18.60 & 13.67 & 13.33 & 29.87 & 29.02 & 7.53 & 109.99 \\
\hline 34 & BML $2 \times$ CML 124 & 68.67 & 71.67 & 3.00 & 101.33 & 50.00 & 178.21 & 82.78 & 2.84 & 209.53 & 41.87 & 18.53 & 14.27 & 12.80 & 32.13 & 29.81 & 9.12 & 105.81 \\
\hline 35 & BML $6 \times$ BML 7 & 68.00 & 70.33 & 2.33 & 103.67 & 48.03 & 186.04 & 83.06 & 3.12 & 209.80 & 39.33 & 19.67 & 15.13 & 14.93 & 35.67 & 31.05 & 8.69 & 129.57 \\
\hline 36 & BML $6 \times$ BML 15 & 69.00 & 72.00 & 3.00 & 105.67 & 45.53 & 200.17 & 81.57 & 2.12 & 222.67 & 37.53 & 19.87 & 15.07 & 13.73 & 36.13 & 27.07 & 9.21 & 118.43 \\
\hline 37 & BML $6 \times$ BML 14 & 67.67 & 70.33 & 2.67 & 100.67 & 51.57 & 170.85 & 77.19 & 2.66 & 204.00 & 41.53 & 17.20 & 14.13 & 13.73 & 26.47 & 27.22 & 8.55 & 95.80 \\
\hline 38 & BML $6 \times$ CM 118 & 65.00 & 67.00 & 2.00 & 99.67 & 54.37 & 178.22 & 82.18 & 2.64 & 184.87 & 42.93 & 17.53 & 14.27 & 14.67 & 29.13 & 26.34 & 8.01 & 98.64 \\
\hline 39 & BML $6 \times$ CM 119 & 67.67 & 70.00 & 2.33 & 100.00 & 56.77 & 189.75 & 84.88 & 1.42 & 171.27 & 38.60 & 16.67 & 14.80 & 15.07 & 26.00 & 25.90 & 10.10 & 94.49 \\
\hline 40 & BML $6 \times$ CML 124 & 69.00 & 72.00 & 3.00 & 105.00 & 47.40 & 202.68 & 77.71 & 1.49 & 192.93 & 40.13 & 16.87 & 14.53 & 14.00 & 26.40 & 26.18 & 8.31 & 98.23 \\
\hline 41 & BML $7 \times$ BML 15 & 69.67 & 72.00 & 2.33 & 104.33 & 53.00 & 168.92 & 87.77 & 1.64 & 225.67 & 41.33 & 19.13 & 14.93 & 14.13 & 36.31 & 31.64 & 8.09 & 143.33 \\
\hline 42 & BML $7 \times$ BML 14 & 68.00 & 69.67 & 1.67 & 102.67 & 52.10 & 173.42 & 87.27 & 1.79 & 211.80 & 44.33 & 18.87 & 15.40 & 13.20 & 32.80 & 31.75 & 11.04 & 132.39 \\
\hline 43 & BML 7 × CM 118 & 68.33 & 70.33 & 2.00 & 102.67 & 48.10 & 191.67 & 86.57 & 1.87 & 198.87 & 40.33 & 18.27 & 14.87 & 14.80 & 31.40 & 29.77 & 9.64 & 124.64 \\
\hline 44 & BML $7 \times$ CM 119 & 67.00 & 69.00 & 2.00 & 104.00 & 53.23 & 170.41 & 81.34 & 2.88 & 218.60 & 41.87 & 18.67 & 14.60 & 14.13 & 31.20 & 27.25 & 10.70 & 111.97 \\
\hline 45 & BML 7 × CML 124 & 68.67 & 72.00 & 3.33 & 104.67 & 54.47 & 168.04 & 81.51 & 2.71 & 202.87 & 43.80 & 16.60 & 14.60 & 12.80 & 27.33 & 26.45 & 8.26 & 84.25 \\
\hline 46 & BML $15 \times$ BML 14 & 69.33 & 71.67 & 2.33 & 104.67 & 53.93 & 179.58 & 86.20 & 2.77 & 190.27 & 40.73 & 17.93 & 14.00 & 13.73 & 29.93 & 26.15 & 8.81 & 106.21 \\
\hline 47 & BML $15 \times$ CM 118 & 68.33 & 70.67 & 2.33 & 102.67 & 50.27 & 183.27 & 83.29 & 3.28 & 200.67 & 39.00 & 17.07 & 14.07 & 14.40 & 30.93 & 27.16 & 7.86 & 105.25 \\
\hline 48 & BML $15 \times$ CM 119 & 67.67 & 69.67 & 2.00 & 100.33 & 54.00 & 164.75 & 89.11 & 2.71 & 181.07 & 38.87 & 17.13 & 14.60 & 14.80 & 27.67 & 26.87 & 8.18 & 98.94 \\
\hline 49 & BML $15 \times$ CML 124 & 68.33 & 70.33 & 2.00 & 100.67 & 52.70 & 169.89 & 77.52 & 3.66 & 224.07 & 40.93 & 19.00 & 14.73 & 13.20 & 29.73 & 27.56 & 9.77 & 97.22 \\
\hline 50 & BML $14 \times$ CM 118 & 68.67 & 71.33 & 2.67 & 101.33 & 51.00 & 169.23 & 85.38 & 1.38 & 197.13 & 37.80 & 15.80 & 13.67 & 14.00 & 23.00 & 23.31 & 7.35 & 76.96 \\
\hline 51 & BML $14 \times$ CM 119 & 66.00 & 68.00 & 2.00 & 100.00 & 49.40 & 168.43 & 83.80 & 1.90 & 189.40 & 41.73 & 17.93 & 14.13 & 13.07 & 27.60 & 24.45 & 8.54 & 89.42 \\
\hline 52 & BML $14 \times$ CML 124 & 68.33 & 70.67 & 2.33 & 99.67 & 53.60 & 160.62 & 80.61 & 1.74 & 208.93 & 45.87 & 17.93 & 15.00 & 13.20 & 29.00 & 27.50 & 8.63 & 107.27 \\
\hline 53 & CM $118 \times$ CM 119 & 68.00 & 69.33 & 1.33 & 100.33 & 54.57 & 168.22 & 84.91 & 2.82 & 183.47 & 38.73 & 16.67 & 13.40 & 13.47 & 28.07 & 25.42 & 8.14 & 83.81 \\
\hline 54 & CM $118 \times$ CML 124 & 68.00 & 70.00 & 2.00 & 100.00 & 54.43 & 196.14 & 80.03 & 3.03 & 235.93 & 43.40 & 18.60 & 14.27 & 13.33 & 27.93 & 26.89 & 8.91 & 90.72 \\
\hline \multirow[t]{7}{*}{55} & CM $119 \times$ CML 124 & 66.67 & 68.33 & 1.67 & 99.33 & 54.67 & 162.37 & 78.86 & 2.32 & 220.00 & 40.20 & 18.27 & 15.07 & 13.73 & 27.47 & 28.08 & 8.19 & 93.13 \\
\hline & Grand mean & 68.02 & 70.37 & 2.35 & 102.42 & 50.58 & 177.12 & 84.36 & 2.34 & 208.88 & 40.41 & 18.52 & 14.50 & 13.60 & 29.51 & 27.52 & 8.90 & 103.57 \\
\hline & Range (Min.) & 65.00 & 67.00 & 1.33 & 98.67 & 38.91 & 159.85 & 71.03 & 1.11 & 171.27 & 35.13 & 15.80 & 13.33 & 12.13 & 21.13 & 19.77 & 7.35 & 76.17 \\
\hline & Range (Max.) & 71.33 & 73.67 & 3.33 & 107.33 & 56.77 & 205.75 & 90.79 & 3.66 & 235.93 & 45.87 & 20.93 & 15.80 & 15.07 & 36.31 & 31.84 & 11.04 & 143.33 \\
\hline & C.D. & 1.47 & 1.88 & 0.63 & 1.48 & 3.56 & 19.88 & 4.85 & 0.19 & 15.26 & 4.11 & 1.50 & 0.90 & 1.06 & 3.20 & 1.59 & 0.15 & 7.22 \\
\hline & S.E(m) & 0.53 & 0.67 & 0.38 & 0.53 & 1.27 & 7.08 & 1.73 & 0.07 & 5.44 & 1.47 & 0.53 & 0.32 & 0.38 & 1.14 & 0.57 & 0.05 & 2.57 \\
\hline & C.V. & 1.34 & 1.65 & 22.60 & 0.89 & 4.34 & 6.93 & 3.55 & 4.91 & 4.51 & 6.28 & 5.00 & 3.81 & 4.81 & 6.69 & 3.56 & 1.03 & 4.30 \\
\hline
\end{tabular}

DFT - Days to 50\% Tasseling, DFS - Days to 50\% Silking, ASI - Anthesis Silking Interval, DM - Days to Maturity, SCMR - SPAD Chlorophyll Meter

Reading SLA - Specific Leaf Area $\left(\mathrm{cm}^{2} \mathrm{~g}^{-1}\right)$, RWC - Relative Water Content (\%), LAI - Leaf Area Index, PH - Plant Height (cm), TL - Tassel Length (cm), EL - Ear Length (cm), EG - Ear Girth (cm), KR/E - No. of Kernel Rows per Ear, K/ER - No. of Kernels per Ear Row, 100 KW - 100 Kernel Weight (g), PC -

Protein Content (\%), KYD - Kernel Yield per Plant (g) 
Table.5 Top five promising hybrids identified based on mean performance for yield and yield components in maize under rice fallow situations

\begin{tabular}{|c|c|c|c|c|c|}
\hline S. No. & Character & Top five hybrids & S. No. & Character & Top five hybrids \\
\hline 1 & $\begin{array}{l}\text { Days to } 50 \% \\
\text { tasseling }\end{array}$ & $\begin{array}{l}\text { BML } 6 \times \text { CM } 118 \\
\text { BML } 51 \times \text { BML } 2 \\
\text { BML } 5 \times \text { BML } 14 \\
\text { BML } 2 \times \text { CM } 119 \\
\text { BML } 14 \times \text { CM } 119\end{array}$ & 10 & Tassel length $(\mathrm{cm})$ & $\begin{array}{l}\text { BML } 14 \times \text { CML } 124 \\
\text { BML } 5 \times \text { BML } 14 \\
\text { BML } 5 \times \text { CM } 119 \\
\text { BML } 7 \times \text { BML } 14 \\
\text { BML } 7 \times \text { CML } 124\end{array}$ \\
\hline 2 & Days to $50 \%$ silking & $\begin{array}{l}\text { BML } 6 \times \text { CM } 118 \\
\text { BML } 51 \times \text { BML } 2 \\
\text { BML } 5 \times \text { BML } 14 \\
\text { BML } 2 \times \text { CM } 119 \\
\text { BML } 14 \times \text { CM } 119\end{array}$ & 11 & Ear length $(\mathrm{cm})$ & $\begin{array}{l}\text { BML } 5 \times \text { BML } 2 \\
\text { BML } 5 \times \text { BML } 15 \\
\text { BML } 5 \times \text { BML } 14 \\
\text { BML } 5 \times \text { BML } 7 \\
\text { BML } 5 \times \text { BML } 6\end{array}$ \\
\hline 3 & $\begin{array}{l}\text { Anthesis silking } \\
\text { interval }\end{array}$ & $\begin{array}{l}\text { CM } 118 \times \text { CM } 119 \\
\text { BML } 7 \times \text { BML } 14 \\
\text { CM } 119 \times \text { CML } 124 \\
\text { BML } 51 \times \text { BML } 6 \\
\text { BML } 51 \times \text { BML } 7\end{array}$ & 12 & Ear girth $(\mathrm{cm})$ & $\begin{array}{l}\text { BML } 51 \times \text { BML } 7 \\
\text { BML } 5 \times \text { CM } 105 \\
\text { BML } 7 \times \text { BML } 14 \\
\text { BML } 5 \times \text { BML } 6 \\
\text { CM } 105 \times \text { BML } 15\end{array}$ \\
\hline 4 & Days to maturity & $\begin{array}{l}\text { BML } 5 \times \text { BML } 15 \\
\text { BML } 5 \times \text { BML } 7 \\
\text { BML } 2 \times \text { BML } 15 \\
\text { CM } 119 \times \text { CML } 124 \\
\text { BML } 6 \times \text { CM } 118 \\
\text { BML } 14 \times \text { CML } 124\end{array}$ & 13 & $\begin{array}{l}\text { No. of kernel } \\
\text { rows/ear }\end{array}$ & $\begin{array}{l}\text { BML } 6 \times \text { CM } 119 \\
\text { BML } 6 \times \text { BML } 7 \\
\text { BML } 7 \times \text { CM } 118 \\
\text { BML } 15 \times \text { CM } 119 \\
\text { BML } 6 \times \text { CM } 118\end{array}$ \\
\hline 5 & $\begin{array}{l}\text { SPAD Chlorophyll } \\
\text { Meter Reading }\end{array}$ & $\begin{array}{l}\text { BML } 6 \times \text { CM } 119 \\
\text { BML } 51 \times \text { CML } 124 \\
\text { BML } 51 \times \text { BML } 7 \\
\text { CM } 119 \times \text { CML } 124 \\
\text { CM } 118 \times \text { CM } 119\end{array}$ & 14 & $\begin{array}{l}\text { No. of kernels/ear } \\
\text { row }\end{array}$ & $\begin{array}{l}\text { BML } 7 \times \text { BML } 15 \\
\text { BML } 6 \times \text { BML } 15 \\
\text { BML } 5 \times \text { BML } 15 \\
\text { BML } 6 \times \text { BML } 7 \\
\text { BML } 2 \times \text { BML } 7\end{array}$ \\
\hline 6 & $\begin{array}{l}\text { Specific leaf area } \\
\left(\mathrm{cm}^{2} \mathrm{~g}^{-1}\right)\end{array}$ & $\begin{array}{l}\text { BML } 51 \times \text { BML } 15 \\
\text { BML } 5 \times \text { CM } 105 \\
\text { BML } 14 \times \text { CML } 124 \\
\text { CM } 105 \times \text { BML } 6 \\
\text { BML } 51 \times \text { CM } 118\end{array}$ & 15 & $\begin{array}{l}100 \text { kernel weight } \\
(\mathrm{g})\end{array}$ & $\begin{array}{l}\text { BML } 51 \times \text { BML } 7 \\
\text { BML } 7 \times \text { BML } 14 \\
\text { BML } 7 \times \text { BML } 15 \\
\text { BML } 51 \times \text { CM } 118 \\
\text { BML } 2 \times \text { BML } 7\end{array}$ \\
\hline 7 & $\begin{array}{l}\text { Relative water } \\
\text { content }(\%)\end{array}$ & $\begin{array}{l}\text { BML } 5 \times \text { BML } 6 \\
\text { BML } 2 \times \text { BML } 7 \\
\text { CM } 105 \times \text { BML } 2 \\
\text { BML } 51 \times \text { BML } 6 \\
\text { CM } 105 \times \text { BML } 14\end{array}$ & 16 & Protein content $(\%)$ & $\begin{array}{l}\text { BML } 7 \times \text { BML } 14 \\
\text { BML } 5 \times \text { BML } 14 \\
\text { BML } 7 \times \text { CM } 119 \\
\text { BML } 5 \times \text { CM } 119 \\
\text { BML } 51 \times \text { BML } 2\end{array}$ \\
\hline 8 & Leaf area index & $\begin{array}{l}\text { BML } 15 \times \text { CML } 124 \\
\text { CM } 105 \times \text { CM } 119 \\
\text { BML } 2 \times \text { CM } 119 \\
\text { BML } 15 \times \text { CM } 118 \\
\text { CM } 105 \times \text { BML } 14\end{array}$ & 17 & $\begin{array}{l}\text { Kernel yield per } \\
\text { plant }(\mathrm{g})\end{array}$ & $\begin{array}{l}\text { BML } 7 \times \text { BML } 15 \\
\text { BML } 2 \times \text { BML } 7 \\
\text { BML } 7 \times \text { BML } 14 \\
\text { BML } 6 \times \text { BML } 7 \\
\text { BML } 7 \times \text { CM } 118\end{array}$ \\
\hline 9 & Plant height $(\mathrm{cm})$ & $\begin{array}{l}\text { CM } 118 \times \text { CML } 124 \\
\text { BML } 51 \times \text { CM } 118 \\
\text { BML } 51 \times \text { BML } 2 \\
\text { BML } 5 \times \text { BML } 7 \\
\text { BML } 5 \times \text { BML } 6\end{array}$ & & & \\
\hline
\end{tabular}


The mean sum of squares due to genotypes (parents and hybrids) was highly significant for all the traits studied, there by indicating the existence of sufficient variability in the material studied.

In any plant breeding programme for developing high yielding hybrids or varieties, the basic need is the choice of parents with high mean values as they are expected to produce desirable segregants upon crossing (Gilbert, 1958). In the present investigation several parents showed high mean performance for more than one character (Table 3). Among the eleven parents, the parent BML 2 exhibited high per se performance for seven traits viz., kernel yield per plant, 100 kernel weight, number of kernels per ear row, ear length, plant height, tassel length and days to $50 \%$ tasseling and it is also exhibited good per se performance for protein content, ear girth, leaf area index, SCMR and days to $50 \%$ silking and hence adjudged as best parent among the eleven parents.

Next to this the parent BML 14 showed high per se performance for three traits viz., number of kernel rows per ear, leaf area index and days to $50 \%$ tasseling and it also showed good per se performance for kernel yield per plant, 100 kernel weight, ear length, ear girth, plant height, tassel length, SCMR and days to $50 \%$ silking.

The parent BML 6 exhibited good per se performance for kernel yield per plant, ear girth, ear length, number of kernel rows per ear, number of kernels per ear row, plant height, tassel length and leaf area index, while the parents CM 105, CM 118 and CM 119 were found to be the early inbred lines since they exhibited early anthesis, early silking, less anthesis-silking interval and early maturity. Hence it could be suggested that selection of these parents viz., BML 2, BML
14, BML 6, CM 105, CM 118 and CM 119 in hybridization programme would be effective for improvement of maturity, yield and yield components under rice fallow situation.

Based on mean performance of hybrids it could be revealed that no single cross hybrid was found significant for all yield and yield component traits (Table 4). The top five promising hybrids identified for yield and various yield component traits are presented in Table 5. Among the 55 hybrids, the hybrid BML $7 \times$ BML 15 recorded high per se performance for kernel yield per plant followed by BML $2 \times$ BML 7, BML $7 \times$ BML 14, BML $6 \times$ BML 7 and BML $7 \times$ CM 118. Besides kernel yield, the hybrids BML 7 $\times$ BML 15 and BML $2 \times$ BML 7 also recorded good per se performance for number of kernels per ear row, 100 kernel weight. Similarly the hybrid BML $7 \times$ BML 14 also recorded good per se performance for 100 kernel weight, protein content, ear girth, tassel length and anthesis silking interval besides kernel yield. Likewise, the hybrid BML $6 \times$ BML 7 showed good per se performance for number of kernel rows per ear, number of kernels per ear row, 100 kernel weight and ear girth besides kernel yield. The hybrid BML 7 $\times$ CM 118 showed good per se performance for ear girth and number of kernel rows per ear besides kernel yield. Hence, selection would be effective for these five crosses as they exhibited good per se performance for yield and most of the yield components. It was interesting to note that in all these superior hybrids BML 7 was one of the parents which indicated that this parent might be the best general combiner and could be included in future breeding programmes. Similarly, Naik (2012), Karki and Shrestha (2014), Rao et al., (2016) and Govardhanrao and Ramana (2017) also conducted similar studies under rice fallow situation and reported the applicability of maize cultivation under rice fallow situation. 
The results obtained in the present investigation are quite encouraging as the hybrids excelled in their performance under rice fallow condition and may offer a vast scope for breeders to work on the genetic studies under such situation. Based on mean performance it could be concluded that the parents BML 2, BML 14 and BML 6 and the hybrids BML $7 \times$ BML 15, BML $2 \times$ BML 7, BML $7 \times$ BML 14, BML $6 \times$ BML 7 and BML $7 \times$ CM 118 were adjudged as the best parents and hybrids respectively, under rice fallow situation.

\section{References}

Directorate of Economics and Statistics, Govt. of Andhra Pradesh. 201617.Agricultural statistics at a glance Andhra Pradesh.

Gilbert, N.E.G. 1958. Diallel cross in plant breeding. Heredity. 12: 477-498.

Govardhanrao, V and Ramana, P.V. 2017. Economic performance of zero tillage technology in maize under agency tracts of Andhra Pradesh. Asian Journal of
Agricultural Extension, Economics and Sociology. 16(4): 1-4.

INDIASTAT. 2016-2017. http://www. Indiastat.com.

Karki, T.B and Shrestha, J. 2014. Maize production under no-tillage system in Nepal. World Journal of Agricultural Research. 2 (6A): 13-17.

Naik, V.S. 2012. Genetic studies for improvement of single cross hybrids of maize (Zea mays L.) through identification of elite inbreds with favourable alleles for yield and yield components. Ph.D. Thesis, Acharya N.G. Ranga Agricultural University, Guntur.

Panse, V.G and Sukhatme. 1985. Statistical methods for Agricultural workers. Published by ICAR, New Delhi.

Rao, P.V., Subbaiah, G and Veeraraghavaiah, R. 2016. Productivity and nutrient uptake of rice fallow maize (Zea mays L.) As influenced by plant density and fertilizer $\mathrm{N}$ under no-till conditions. International Journal of Current Microbiology and Applied Sciences. 5(6): 826-836.

\section{How to cite this article:}

Paramesh, M., K.H.P. Reddy, D.M. Reddy, V. Rajarajeswari and Bhaskara Reddy, B.V. 2018. Evaluation of Maize Genotypes under Rice Fallow Situation. Int.J.Curr.Microbiol.App.Sci. 7(08): 1339-1347. doi: https://doi.org/10.20546/ijcmas.2018.708.152 